\title{
Artificial Microstructure Generation of DP590 steel using multi-objective TLBO methodology
}

\author{
Ashwani Verma and Ravindra K. Saxena*
}

\begin{abstract}
Micromechanical modelling of dual phase (DP) steel is used to predict the macroscopic tensile properties. The DP steel is widely used due to its good formability characteristics. The DP steels are having high strength and high ductility for suitable application in automobile industries. For the micromechanical analysis of the DP steel, the representative volumetric element (RVE) is generated from the microstructure of the steel obtained using scanning electron microscope at a suitable magnification showing explicit ferrite and martensite grains. In the present work, a model is formulated using TeacherLearner based optimization (TLBO) algorithm to generate the artificial microstructure of the DP590 steel. The micromechanical modeling is performed to get the tensile macroscopic response. The model is able to predict the artificial microstructure and the tensile stress-strain of the DP590 steel with a reasonable accuracy.
\end{abstract}

Keywords-DP590 steel, micromechanical modeling, artifical microstructure, TLBO, finite element method, MATLAB.

\section{Introduction}

The automobile industry is growing steadily and demand for high strength materials is increasing. The dual phase (DP) steel is generated consisting of ferrite matrix with martensite inclusions. The austenite in the steels is completely transformed to martensite under controlled heat treatment in DP steels. The DP steels have high strength and ductility to be widely used for forming applications to the benefit for the automobiles. The determination of properties of DP steels is widely studied. Due to its increasing demand, a number of researchers [1-4] have attempted to analyze the characteristics of the DP steels using micromechanical modelling. A representative volumetric element (RVE) is generated using the micromechanical image obtained using scanning electron microscope (SEM). Recently, Abid et al. [5] have used random number of generator to generate artificial microstructure. Further, Hou et al. [6] have used Topology optimization to generate RVE for DP590 steel.

\section{Ashwani Verma (Author)}

Department of Mechanical Engineering, Sant Longowal Institute of Engineering and Technology,

Longowal-148106, District Sangrur, Punjab, INDIA

Ravindra K. Saxena (Author)

Department of Mechanical Engineering, Sant Longowal Institute of Engineering and Technology,

Longowal-148106, District Sangrur, Punjab, INDIA
In this work, an attempt has been made to generate the artificial microstructure of DP590 steel using Voronoi tessellation and Teacher-Learner based Optimization method. The generated RVE model is analyzed for the prediction of macroscopic tensile properties of the DP steel.

\section{Formulation of the model}

\section{A. Teaching-Learning based optimization}

Optimization techniques are the popular methods to find the optimum solution for constrained problem or the maxima or minima of a function. Teaching-learning-based optimization (TLBO) [7] is a population based optimization methodology. The methodology assumes a population which is considered as one class of teachers or a group of learners in a class. In TLBO, different design variables correspond to different subjects offered to learners and the result of learners is treated as 'fitness' [7]. The process for TLBO is divided into two main phases:

Teacher Phase: It is the first part of algorithm where learners learn through the teacher. During this phase, a teacher tries to increase the mean result of the class in the subject taught by him or her depending on his or her capability. The difference between the existing mean result of each subject is given by

$$
M D_{j l i}=r_{i}\left(X_{j, l . b e s t, i}-T_{F} M_{j i}\right)
$$

where, $M_{j i}$ is the mean result of the learner in a particular subject ' $j$ ' in a particular iteration ' $i$ ', $X_{j, l . b e s t, i}$ is the result of the best learner in $j^{\text {th }}$ subject in $i^{\text {th }}$ iteration. $T_{F}$ is the teaching factor which decides the value of mean to be changed, and $r_{i}$ is the random number in the range $(0,1)$. Value of $T_{F}$ can be either 1 or 2 . The value of $T_{F}$ is decided randomly with equal probability and $T_{F}$ is not a parameter of the TLBO algorithm. Based on the $M D_{j l i}$ the existing solution is updated in the teacher phase according to the following expression:

$$
X_{j, l, i}^{\prime}=X_{j, l, i}+M D_{j l i}
$$

where, $X_{j, l, i}^{\prime}$ is the updated value of $X_{j, l, i}$. All the accepted function values at the end of the teacher phase are maintained and these values become input to the learner phase. 
Learner Phase: The learner phase depends upon the teacher phase. It is the second part of the algorithm where learners increase their knowledge by interacting among themselves. A learner interacts randomly with other learners for enhancing his or her knowledge. In this phase, two learners are randomly selected $\mathrm{P}$ and $\mathrm{Q}$ such that $X_{\text {total }-P, i}^{\prime} \neq X_{\text {total }-Q, i}$ (where, $X_{\text {total }-P, i}^{\prime}$ and $X_{\text {total }-Q, i}^{\prime}$ are the updated functional values of $\mathrm{P}$ and $\mathrm{Q}$, respectively, at the end of teacher phase).

$$
X_{j, P, i}^{\prime \prime}=X_{j, P, i}^{\prime}+a b s\left(r_{i}\left(X_{j, P, i}^{\prime}-X_{j, Q, i}^{\prime}\right)\right)
$$

$X_{j, P, i}^{\prime \prime}$ is accepted if it gives a better function values [7].

\section{B. Finite Element Formulation}

For the analysis of the micromechanical model, an inhouse finite element model on MATLAB is developed. The model is based on updated Lagrangian formulation. The governing equations for the model consist of (i) incremental strain-displacement relations, (ii) incremental stress-strain relations, (iii) incremental equations of motion.

The incremental form of the equation of motion is not found to be convenient for the finite element formulation. Instead, an integral form of the equilibrium equation at time is used. It is given by the following virtual work expression [8]:

$$
\int^{t+\Delta t} V{ }^{t+\Delta t} \sigma_{i j} \delta\left({ }^{t+\Delta t} \varepsilon_{i j}\right) d{ }^{t+\Delta t} V={ }^{t+\Delta t} R
$$

Here, ${ }^{t+\Delta t} V$ is the domain, ${ }^{t+\Delta t} R$ is the virtual work of the external forces and ${ }^{t+\Delta t} \sigma_{i j}$ the Cauchy stress tensor, all at time. Further, $\delta\left({ }^{t+\Delta t} \varepsilon_{i j}\right)$ represents the virtual linear strain tensor corresponding to the virtual displacement vector $\delta u_{i}$ at time. Equation (4) is not known in the updated configuration and therefore, it is transformed to ${ }^{t} V$ domain at time $(t)$ using a standard procedure [8]. After, linearization and approximation the final equation is

$$
\begin{array}{r}
\int_{{ }^{t} V}{ }^{t} C_{i j k l}^{E P}{ }_{t} \Delta \varepsilon_{k l} \delta\left({ }_{t} \Delta \varepsilon_{i j}\right) d{ }^{t} V+\int_{{ }_{t}}{ }^{t} \sigma_{i j} \delta\left({ }_{t} \Delta \eta_{i j}\right) d{ }^{t} V \\
+\int_{{ }_{t} V}{ }^{t} \sigma_{i j} \delta\left({ }_{t} \Delta \varepsilon_{i j}\right) d^{t} V={ }^{t+\Delta t} R
\end{array}
$$

where, $C_{i j k l}^{E P}$ is fourth order elasto-plastic constitutive tensor [9], and

$$
{ }_{t} \Delta \varepsilon_{i j}=\left(\frac{1}{2}\right)\left({ }_{t} \Delta u_{i, j}+{ }_{t} \Delta u_{j, i}\right),{ }_{t} \Delta \eta_{i j}=\left(\frac{1}{2}\right){ }_{t} \Delta u_{k, i t} \Delta u_{k, j} \text {. }
$$

The domain is divided into 3-D hexahedral finite elements using the standard shape functions [8]

$$
{ }_{t}\{\Delta u\} \equiv\left\{{ }_{t} \Delta u_{x} \quad{ }_{t} \Delta u_{y} \quad{ }_{t} \Delta u_{z}\right\}^{T}={ }^{t}[\Phi]_{t}\{\Delta u\}^{e}
$$

Here, the vector ${ }_{t}\{\Delta u\}^{e}$ contains the nodal values of the incremental displacement components at the elemental nodes and the matrix ${ }^{t}[\Phi]$ contains the shape functions that are known functions of the coordinates. Substitution of equation (7) into the virtual work expression (5) and assembly over all the elements, leads to the following finite element equation:

$$
{ }^{t}[K]{ }_{t}\{\Delta u\}+{ }^{t}\{f\}={ }^{t+\Delta t}\{F\} . t+\Delta t
$$

Here, ${ }_{t}\{\Delta u\}$ is called as the global (incremental) displacement vector and ${ }^{t}[K],{ }^{t}\{f\}$ and ${ }^{t+\Delta t}\{F\}$, respectively, denote the global stiffness matrix (at time $t$ ), global internal force vector (at time $t$ ) and global external force vector (at time $t+\Delta t$ ). The expressions for these matrices are available in any standard text book on non-linear FEM [8].

\section{Microstructure generation model}

Voronoi tessellation allows the generation of artificial microstructures with randomly distributed and orientated grains for metallic materials [10]. The tessellation is generated based on random seeds within a defined domain. The microstructure is generated and analyzed to maintain the periodicity. The seed points are generated based on random number generator. The generation of the microstructural model should be independent of the sequence of the random number generator. For the present work, the microstructure of the dual phase steel is generated based on the inbuilt default random number generator sequence in MATLAB.

The microstructure of the dual phase steel to consists of martensite and ferrite matrix. It is assumed that the microstructure has martensite inclusion in a ferrite matrix. The DP steel is distinguished based on the volume fraction of the martensite in the domain. It is reported that the connectivity of the martensite within the ferrite matrix in a dual phase steel is an important parameter determining the tensile behavior [11]. The connectivity is defined as $\varphi=\phi_{F M} /\left(\phi_{F M}+\phi_{F F}\right)$, where $\phi_{F M}$ is the number of ferrite/martensite boundaries per unit length and $\phi_{F F}$ is the number of ferrite/ferrite boundaries per unit length (excluding martensite boundaries). In view that the model for generating the artificial microstructure is formulated as a multi-objective optimization problem with following objective functions:

$$
\Omega=\frac{\text { total area of the martensite grains }}{\text { total area of domain }}=\frac{a^{T} \rho}{a^{T} I}
$$

where, $\Omega$ is the volume fraction of the martensite, $a$ is a vector containing the area of each of the cells in a voronoi diagram, $\rho$ is the vector containing the values assigned to 
ferrite and martensite islands/grain/cells within the range $(0,1)$ with ' 0 ' for the ferrite and ' 1 ' for the martensite cells and $I$ is the identity vector. For the optimization problem, the total area of the domain is assumed to be equal to 1 . The other objective function i.e., connectivity of the martensite in ferrite matrix is given by

$$
\varphi=\frac{\phi_{F M}}{\left(\phi_{F M}+\phi_{F F}\right)}
$$

where, all the associated terms are already defined. The objective function for the optimization problem is formulated as:

$$
\min f=c_{1} * \operatorname{abs}\left(\Omega-\Omega_{\text {target }}\right)+c_{2} * \operatorname{abs}\left(\varphi-\varphi_{\text {arget }}\right)
$$

subjected to

$$
\begin{aligned}
& \rho=\left\{\begin{array}{l}
0, \text { for } \Omega_{F} \\
1, \text { for } \Omega_{M}
\end{array}\right. \\
& \forall \rho \in(0,1)
\end{aligned}
$$

where, $c_{1}$ and $c_{2}$ are weight fractions, $\Omega_{F}$ is the area/volume element assigned for ferrite grain, $\Omega_{M}$ is the area/volume element assigned for martensite grains, $\Omega_{\text {target }}$ is the targeted volume fraction of martensite in the domain, and $\varphi_{\text {arget }}$ is the targeted martensite connectivity. For the generation of the artificial microstructure, values of both the fractions, $c_{1}$ and $c_{2}$ are taken to be equal. Further, following constraint is maintained:

$$
\begin{aligned}
& \Omega_{\mathrm{F}} \cap \Omega_{\mathrm{M}}=\varnothing, \text { and } \\
& \Omega_{\mathrm{F}} \cup \Omega_{\mathrm{M}}=\Omega_{T}
\end{aligned}
$$

where, $\Omega_{T}$ is the total area/volume of the microstructure.

\section{Results}

The macroscopic response for the dual phase steel is obtained using the micromechanical model. The tensile behavior of the dual phase steel is dependent on the volume fraction of the martensite. The connectivity of the martensite is another factor which affect the stress-strain characteristics. The micrograph of the DP590 steel obtained from Paul [4] is shown in Fig. 1. Dark gray spots reveal the martensite grains and light grey large sized zone depicts the ferrite grain the micrograph. It is observed that there are small grains of martensite embedded in the ferrite matrix. The volume fraction of martensite is $10 \%$ in this DP590 steel. The analysis of the micrograph reveals that the connectivity of the martensite is found to be 0.2 .

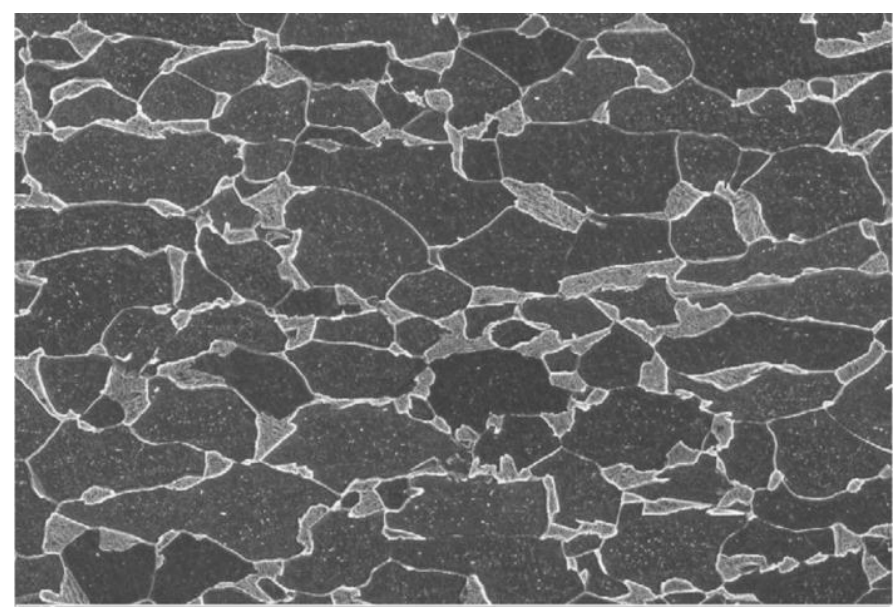

Figure 1. Scanning electron micrograph of DP590 steel at $5.00 \mathrm{KX}$ magnification [4]

The model developed in Section II is applied to predict the typical artificial micrograph of the DP590 steel. The targeted volume fraction $\Omega_{\text {target }}$ is 0.10 and targeted connectivity of the martensite $\varphi_{\text {arget }}$ is 0.20 . The model developed based on TLBO methodology is applied with termination criterion that both the objective functions are satisfied. The value of the constants $c_{1}$ and $c_{2}$ is taken to be 0.5 each.

The generated artificial micrograph obtained from the present model is given Fig. 2. The white color spots depict the martensite and black color domain is ferrite matrix. A total of 1500 random seed points based on default random number generator sequence in MATLAB is taken to generate the micrograph. The class population is taken to be 100. Further, the obtained micrograph is checked for different class strength of the students as per the algorithm of TLBO methodology. It is observed that there is minimal deviation in the predicted micrograph if there is large deviation in the assumed strength of the class. The algorithm is generated using the optimization criterion to ensure that the generated microstructure should not be depended on the different algorithm of the random number sequence generator.

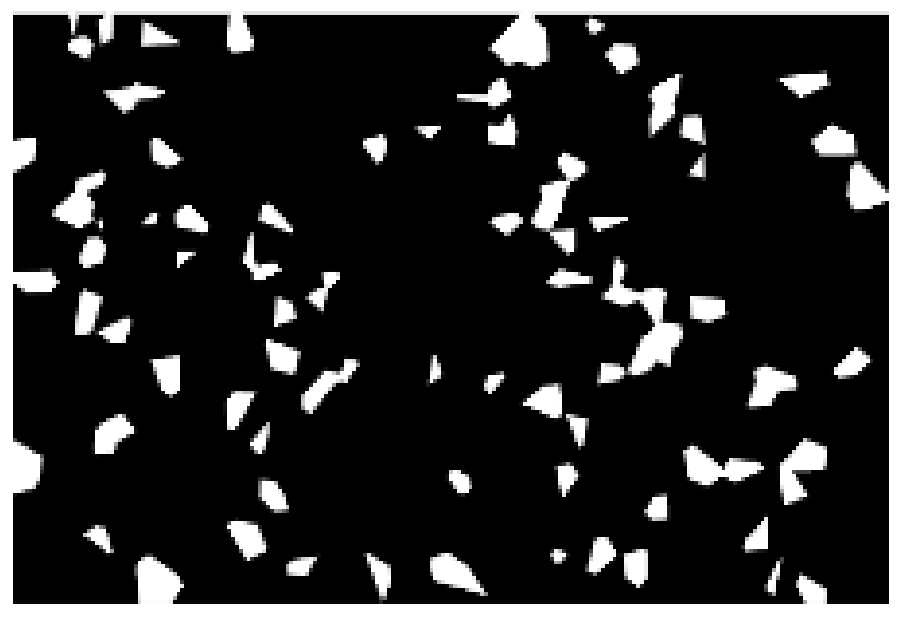

Figure 2. Predicted artificial microstructure of DP590 steel 


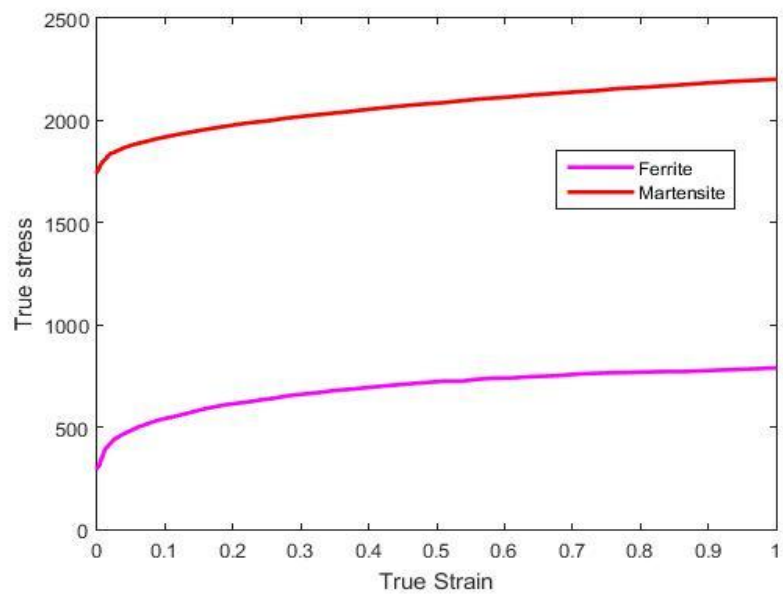

Figure 3. True stress and True strain curve for Ferrite and Martensite

The artificial micrograph obtained in Fig. 2 is discretized using iso-parametric mesh. The effect of mesh size in the range $0.1 \mu \mathrm{m}$ to $2 \mu \mathrm{m}$ are investigated by Ramazani et al. [12]. It is found that the element length smaller than $0.25 \mu \mathrm{m}$ have a negligible effect on the tensile properties obtained from micro-mechanical analysis. It is found that the element size smaller than $0.25 \mu \mathrm{m}$ have a negligible effect on the representative volume element (RVE) in Fig. 2 is discretized using hexahedral element with element length of $0.25 \mu \mathrm{m}$ in the plane of the micrograph with $1 \mu \mathrm{m}$ along the thickness direction. Only one element is taken along the thickness directions. The RVE is assumed to deform only in the plane and all the out-of-plane degree of freedom (DOF) for all the nodes are fixed.

The tensile properties for ferrite and martensite of DP590 steel obtained from reference [4] are given Fig. 3. The periodic grain fragments that intersect the RVE's surface are assumed as a single grain to maintain the periodicity of the microstructure.

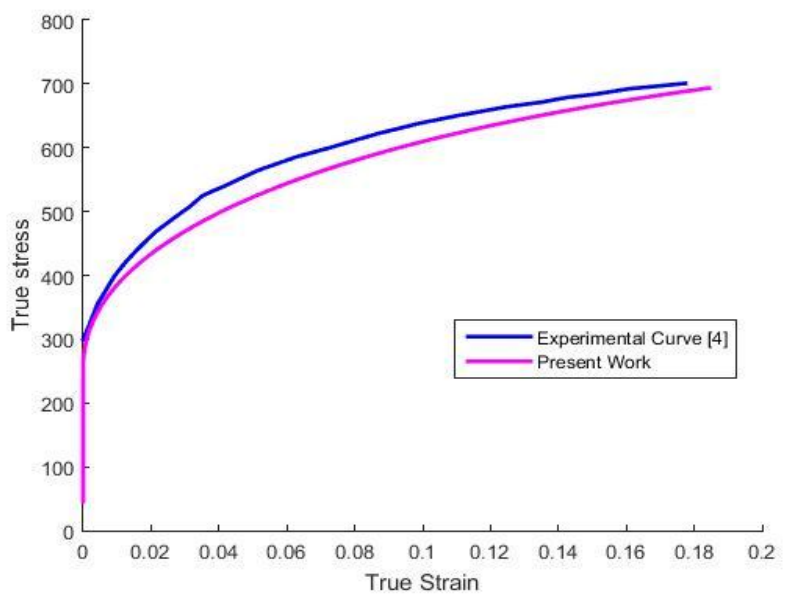

Figure 4. True stress-True strain cure of DP590 steel

The predicted flow curve obtained from the finite element analysis of the RVE generated from the artificial micrograph is given in Fig. 4. For the comparison purpose the experimental flow curve of DP590 steel [4] is also plotted. It is observed that the tensile stress-strain curve of the macroscale for DP590 steel obtained using homogenization methodology $[10]$ is in reasonable agreement.

\section{Conclusions}

The artificial microstructure of DP590 steel is generated using multi-objective optimization technique. The TeacherLearner based optimization (TLBO) technique is effectively employed to predict the micrograph of the steel. The volume fraction of martensite and connectivity of the martensite are the parameters used for the definition of the objective function along with the constraint of the pixel definition of ferrite and martensite for optimization problem. The artificial micrograph obtained from the proposed model is applied to predict the macroscopic tensile stress-strain curve of the dual phase DP590 steel using micromechanical modelling technique. There is a reasonable agreement between the predicted and experimental tensile stress-strain curve. It is concluded that the model is able to effectively predict the artificial micrograph of the DP590 steel and the resulting stress-strain characteristics.

\section{References}

[1] F.M. Al-Abbasi and J.A. Nemes, Micromechanical modeling of dual phase steels, International Journal of Mechanical Sciences, vol. 45, 2003, pp. 1449-1465.

[2] A. Ramazani, K. Mukherjee, U. Prahl, W. Bleck, Transformationinduced geometrically necessary dislocation-based flow curve modelling of dual-phase steels: Effect of grain size, Metallurgical and Materials Transactions A, vol. 43 (10), 2012, pp. 3850-3869.

[3] V. Uthaisangsuk, U. Prahl, and W. Bleck, Characterisation of formability behaviour of multiphase steels by micromechanical modelling, International Journal of Fracture, vol. 157, 2009, pp. 55-69.

[4] S. K. Paul, Real microstructure based micromechanical model to simulate microstructural level deformation behavior and failure initiation in DP 590 steel, Materials \& Design, vol. 44, 2013, pp. 397-406.

[5] N. H. Abid, R. K. Abu Al-Rub, A. N. Palazotto, Computational modeling of the effect of equiaxed heterogeneous microstructures on strength and ductility of dual phase steels, In Computational Materials Science, vol. 103, 2015, pp. 20-37.

[6] Y. Hou, T. Sapanathan, A. Dumon, P. Culière, M. Rachik, A novel artificial dual-phase microstructure generator based on topology optimization, In Computational Materials Science, vol. 123, 2016, pp. 188-200

[7] R.V. Rao, Teaching Learning Based Optimization Algorithm and Its Engineering Applications, Springer, 2016.

[8] K.J. Bathe, Finite Element Procedures, Printeice Hall, 1996

[9] P.M. Dixit, U.S. Dixit, Plasticity: Fundamentals and Applications, CRC Press, 2017.

[10] L. Madej, J. Wang, K. Perzynski, P.D. Hodgson, Numerical modeling of dual phase microstructure behavior under deformation conditions on the basis of digital material representation, Computational Materials Science, vol. 95, 2014, pp. 651-662

[11] M. Sarwar, T. Manzoor, E. Ahmad, N. Hussain, The role of connectivity of martensite on the tensile properties of a low alloy steel, Materials \& Design, vol. 28(6), 2007, pp. 1928-1933.

[12] A. Ramazani, K. Mukherjee, H. Quade, U. Prahl, W. Bleck, Correlation between 2D and 3D flow curve modelling of DP steels using a microstructure-based RVE approach, Materials Science and Engineering: A, vol. 560, 2013, pp. 129-139. 